\title{
Statistical analysis and stochastic modelling of boundary layer wind speed
}

\author{
T. Laubrich ${ }^{\mathrm{a}}$ and H. Kantz \\ Max-Planck-Institute for the Physics of Complex Systems, Noethnitzer Str. 38, \\ 01187 Dresden, Germany
}

\begin{abstract}
We study the statistics of the horizontal component of atmospheric boundary layer wind speed and introduce a stochastic process which has similar properties. Motivated by the non-stationarity of wind velocity data, we describe statistical methods to verify the picture of natural atmospheric boundary layer turbulence to be composed of successively occurring close to ideal turbulence phases with different parameters. We focus on the fluctuation of wind speed around its mean behaviour and show that there is linear relationship between the standard deviation of the fluctuation and the current mean wind speed. Additionally, we analyse the increment statistics and investigate the time dependence of the parameters describing the increment distribution. Noting that the first order geometric auto regressive process has similar statistical properties, we investigate this similarity and analyse the extent to which this stochastic process is a suitable model for wind speed simulation.
\end{abstract}

\section{Atmospheric boundary layer and wind speed data}

The atmospheric boundary layer (ABL) is the lower part of the atmosphere whose dynamics is more or less strongly influenced by surface effects. Evidently, due to viscosity, the boundary condition is that the wind speed has to decay to zero closely to the surface (non slip boundary conditions). Due to turbulence, this effect is directly visible only in the very few millimeters above the boundary. Under certain conditions which guarantee well mixing of the ABL, the velocity depends logarithmically on the height above ground $[1,2]$. Since real surfaces possess a lot of structure, local wind speeds and in particular their direction can differ strongly from this ideal scenario. Also the vertical motion is trivially suppressed close to any horizontal boundary, so that a turbulent wind field close to a boundary cannot be isotropic. All these effects indeed do not only affect the mean flow, but also the turbulent fluctuations and their statistics. Another important complication is due to buoyancy forces and surface temperatures: The heating of the air by surface contact is much more efficient than the direct heating through solar radiation absorbed in the air. The heating of air masses close to the surface causes instabilities and convective forcing of the air flow, which is particularly relevant on sunny days. This additional forcing contributes strongly to an enhancement of the turbulence intensity. Both effects, surface orography and temperature, cause effectively non-stationary influences, since the first depends on the direction of wind and its mean speed (which both are time-dependent), and the latter depends on solar radiation. Also, they depend on many details of the particular surface. It is evident that detailed realistic modelling of these effects in terms of short time properties of the turbulent flow above a surface is very difficult. Therefore, in this paper we present a statistical analysis of experimental data and a stochastic model for the creation of synthetic wind speed data.

\footnotetext{
a e-mail: laubrich@pks.mpg.de
} 
The goal is to understand the nature of this boundary layer turbulence several tens of meters above ground, as it might be relevant for man-made constructions such as houses, bridges, wind turbines, but also for objects from the biosphere such as trees or the flight of insects. We perform a statistical analysis which is inspired by fully developed laboratory turbulence, but which in addition is suitable to inspect instationarities. Moreover, we propose a stochastic model which is capable of generating synthetic wind speed data, whose statistical properties are similar to experimental boundary layer wind speed. Such models might be useful for the numerical computation of loads on structures, but also for the theoretical exploration of extreme wind gusts.

In this contribution we summarise our results which are/will be published in more detail about wind speed statistics [3] and wind speed modelling [4].

\section{Statistical analysis of wind speed}

In the literature there is ample evidence that the air flow in the ABL is turbulent. E.g., an energy cascade with a power law spectrum $S(k) \approx k^{-5 / 3}$ can typically be observed [2]. Therefore it is reasonable to study wind speed data in the ABL in terms of well established statistics of turbulence. In this section we recall two important statistics: fluctuation and increment statistics.

We analyse data sets of the horizontal component of wind speeds recorded at the Lammefjord site $\mathbf{r}\left(55^{\circ} 47^{\prime} 41^{\prime \prime} \mathrm{N}, 11^{\circ} 26^{\prime} 52^{\prime \prime} \mathrm{E}\right)$ with a frequency of $\nu=8 \mathrm{~Hz}$. The time series is denoted by $\left\{u_{n}\right\}, n=1, \ldots, N$, where $u_{n}^{2}=u_{x}^{2}(t=n / \nu)+u_{y}^{2}(t=n / \nu)$. The wind speed was measured with cup-anemometers mounted on a mast and obtained from [5].

Attention has to be paid due to the non-stationarity of the time series and by that we mean a non-stationarity beyond the turbulent fluctuation. The air flow in the ABL, i.e. in the lowest few hundreds of meters of the atmosphere [6], is strongly influenced by surface roughness and hence orography and land use, but even more by thermal effects through heating from the ground. Both effects do not only introduce additional structures into the turbulent flow, but also cause non-stationarities because these effects depend, e.g. on the intensity of solar radiation and on the direction of the surface wind, which both change much faster than large scale pressure differences which generate the overall wind conditions. That is, there are two sources of non-stationarity: one corresponds to the turbulent fluctuation and is described by parameters such as the turbulence intensity and the other one reflects the temporal change of these parameters due to the change of the external conditions driving the wind. We want to consider this difference in respect to laboratory turbulence with constant driving mechanism in our analysis.

First, we introduce the fluctuation statistics. It is customary in turbulence research $[7,8]$ to decompose the wind speed, in our case the horizontal component, during a period of time $T$ as

$$
u(\mathbf{r}, t)=U(\mathbf{r})+u^{\prime}(\mathbf{r}, t)
$$

where $U(\mathbf{r})$ is the average value of $u(\mathbf{r}, t)$ over the period $T$, which is typically chosen to be $10 \mathrm{~min}$ or $1 \mathrm{~h}$. We will choose a much smaller period in the order of $10 \mathrm{~s}$ in order to improve statistics. The turbulence intensity TI $[2,7]$ is defined as the root mean square of $u^{\prime}(\mathbf{r}, t)$ over the period $T$ in units of $U(\mathbf{r})$ so that it states the percentage of the mean flow which are represented by the velocity fluctuation with frequency larger than $1 / T$. Due to turbulent fluctuation $U(\mathbf{r})$ changes from one period to another, but the TI remains constant and its value only depends on the length $T$ of the periods (the TI increases with increasing $T$ ). The Taylor hypothesis [9], which states that the spatial fluctuations within space $U(\mathbf{r}) T$ can be assessed by temporal fluctuations within period $T$, can be assumed to hold with sufficient accuracy if the corresponding TI is less than $0.5[10]$. As the ABL turbulence depends on the weather situation, the ABL TI can vary with time, i.e., it may only remain constant over some periods of size $T$. We treat it as being constant over $24 \mathrm{~h}$. For a more detailed discussion, the reader is referred to [3].

The fluctuation of the wind speed around the moving average over $m=2 \tilde{m}+1$ sample points with $\tilde{m}=0,1,2, \ldots$ is denoted by

$$
f_{n}^{(m)}=u_{n}-\bar{u}_{n}^{(m)}
$$


where the moving average is given by

$$
\bar{u}_{n}^{(m)}=\frac{1}{m} \sum_{k=-\tilde{m}}^{\tilde{m}} u_{n+k}
$$

That is, the fluctuation reflects the current volatility of the wind speed. Due to the nonstationarity of the time series, a directly derived statistics of the fluctuation is meaningless. But assuming that the fluctuation conditioned on a given value $\bar{u}_{n}^{(m)}=V$ of the moving average behaves stationary, we can estimate the (conditioned) distribution

$$
q^{(m)}(f \mid V)=\left\langle\delta\left(f-f_{n}^{(m)}\right)\right\rangle_{n \in\left\{n: \bar{u}_{n}^{(m)} \in[V-\Delta V / 2, V+\Delta V / 2]\right\}}
$$

for each $24 \mathrm{~h}$ recording individually. Figure 1 depicts the estimated distributions. It can be inferred from the plot that the fluctuation conditioned on $V$ is approximately of a Gaussian distribution with a standard deviation being proportional to $V$. This linear dependence is depicted on top of the boxes in Fig. 1. We are interested in the proportionality factor $\alpha(m)$, which can be interpreted as the TI. Attention has to be paid to the binning of $V$ : if $\Delta V$ is too large, $q^{(m)}(f \mid V)$ corresponds to a superposition of normal distributions with variances ranging from $\alpha(m) \times(V-\Delta V / 2)$ to $\alpha(m) \times(V+\Delta V / 2)$, whereas if $\Delta V$ is too small, there are not enough data points within a $V$ bin to estimate the distribution properly. In order to visualise the result in Fig. 1, $\Delta V$ is chosen to be $0.02,0.5,0.5 \mathrm{~m} \mathrm{~s}^{-1}$ for the data acquired at day 186,191 , and 192 respectively. The proportionality factor $\alpha(m)$, and therefore the slope of the straight line on top of the corresponding box in Fig. 1, is estimated in a way which is independent from the choice of $\Delta V$ by estimating the standard deviation of the set $\left\{f_{n}^{(m)} / \bar{u}_{n}^{(m)}\right\}$. For the three $24 \mathrm{~h}$ recordings it takes the values $0.059,0.094$, and 0.075 , respectively. This analysis shows that the TI is well inside the regime of validity of the Taylor hypothesis and additionally that the turbulence intensity is a well defined number despite fluctuations of $\bar{u}_{n}^{(m)}$. The slight deviations of the standard deviations of $q^{(m)}(f \mid V)$ from linearity in $V$ might be due to a non-constant TI over the considered $24 \mathrm{~h}$ period.

Of course, as the wind speed $u_{n}=\bar{u}_{n}^{(m)}+f_{n}^{(m)}$ cannot be negative the approximation

$$
q^{(m)}(f \mid V) \approx N_{0, \alpha(m)^{2} V^{2}}(f),
$$

where $N_{\mu, \sigma^{2}}(\cdot)$ denotes a Gaussian distribution with mean $\mu$ and variance $\sigma^{2}$, is only valid if the probability for $f<-V$ is negligible. The integral

$$
\int_{-\infty}^{-V} \mathrm{~d} f N_{0, \alpha(m)^{2} V^{2}}(f)=\frac{1}{2}\left[1+\operatorname{erf}\left(-\frac{\sqrt{2}}{2 \alpha(m)}\right)\right]
$$

does not depend on $V$ and is close to zero if $\alpha(m)$ is close to zero-which has been observed at the Lammefjord site.

We compare the result (5) with stationary laboratory turbulence, where from a statistical point of view the estimation of the velocity distribution makes sense. The authors of [11] measured the air speed in an air into air free jet experiment. The air speed is in good approximation of a normal distribution with mean $\bar{u}=2.25 \mathrm{~m} \mathrm{~s}^{-1}$ and standard deviation $0.341 \mathrm{~m} \mathrm{~s}^{-1}$, which corresponds to a TI of 0.15 . As the mean speed does not change with time, the fluctuation corresponds to the air velocity shifted by $\bar{u}$ so that it is normally distributed as well. The parameters of the normal distribution are not that important as they merely reflect the experimental setup, it suffices to note that the fluctuation is normally distributed.

Hence, we can interpret the ABL wind speed time series as a composition of stationary turbulence phases with different mean wind velocities and standard deviation being proportional to the mean wind velocity. The proportionality factor can be treated as being constant over a larger time scale than the scale on which the mean wind speed changes. 

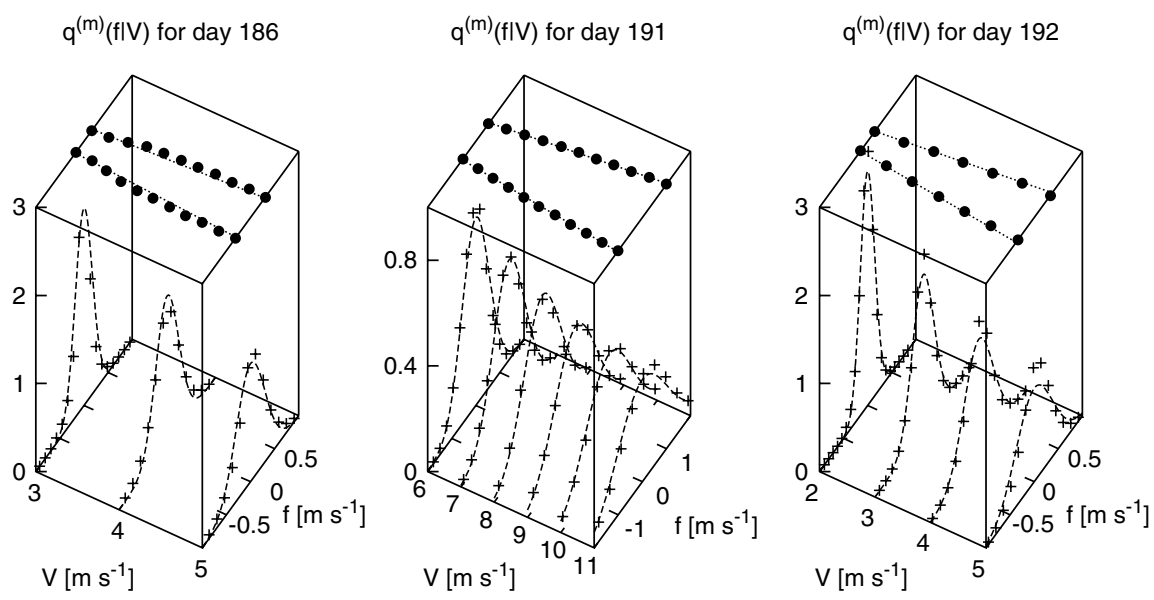

Fig. 1. Estimated distribution of the fluctuation conditioned on the value $V$ of the moving average over $m=101$ time steps for the wind data acquired at the Lammefjord [5] site. The crosses symbolise the estimation whereas the dashed lines represent Gaussian distributions with zero mean and same variance as $q^{(m)}(f \mid V)$. The standard deviation of the fluctuation is again plotted on top of the boxes. The black dots represent the numerical estimation whereas the dotted line corresponds to a line through the origin.

Second, we discuss the increment statistics and verify in detail the hypothesis that natural ABL turbulence is a composition of successively occurring close to ideal turbulence [12-14] with different parameters. The air/wind speed $u(\mathbf{r}, t)$ in ideal turbulence is characterised by its spatial correlations, i.e., the statistics of $u(\mathbf{r}+\Delta \mathbf{r}, t)-u(\mathbf{r}, t)$ which has been hypothesised by Kolmogorov in [14]. The Taylor hypothesis [9] assumes that spatial correlations can be translated into temporal correlations through the transport of spatial structures by the mean flow. Under this assumption, Castaing et al. [15] formulated the following hypothesis for the increment $u(\mathbf{r}, t+\delta t)-u(\mathbf{r}, t)$ : The increment statistics is a superposition of Gaussian distributions with zero mean and variance $1 / \beta$, where $\beta$ is constant on short time intervals. On a larger temporal scale $\beta$ changes according to a log-normal distribution. Assuming that ABL wind speed reflects ideal turbulence, this hypothesis leads to an analytical expression for the distribution of the increment series

$$
u_{s ; n}=u_{n+s}-u_{n}
$$

of the velocity recordings. According to [15] its distribution is given by

$$
\mathrm{Ca}_{\mathrm{s}}\left(u_{s ; n}\right)=\frac{1}{2 \pi \lambda_{s}} \int_{0}^{\infty} \frac{\mathrm{d} \beta \sqrt{\beta}}{\exp }\left[-\frac{1}{2}\left(\frac{1}{\lambda_{s}} \ln \frac{\beta}{\beta_{s}}\right)^{2}-\frac{12}{\beta} u_{s ; n}^{2}\right],
$$

which is referred to as the Castaing distribution. It is described by two parameters $\beta_{s}$ and $\lambda_{s}^{2}$ which are called position and shape parameter, respectively. For positive shape parameter the Castaing distribution is fat-tailed whereas it becomes a Gaussian distribution for $\lambda_{s}^{2}=0$. The $s$-dependence of these parameters is another importants feature of the flow. It has been shown both theoretically [15] and experimentally [16,17] that for ideal turbulence the shape parameters approaches zero for sufficient large $s$. That is, the Castaing distribution takes the shape of a Gaussian distribution with variance $1 / \beta_{s}$ for very large $s$, whereas it resembles a symmetric exponential distribution for small $s$.

We check whether the Lammefjord recordings [5] fulfil the above mentioned hypothesis for ideal turbulence. Instead of testing if the increment distribution for a given increment length $s$ takes the shape of 8 with $\lambda_{s}^{2} \rightarrow 0$ for $s \rightarrow \infty$, we directly test the hypothesis using the superstatistics approach [18]. Assuming that the temporal scale $T_{s}$ where the increment series can be treated as being normally distributed is constant, Beck et al. [19,20] developed an algorithm estimating $T_{s}$. The $\beta$ series corresponds to the inverse of the estimated variance 


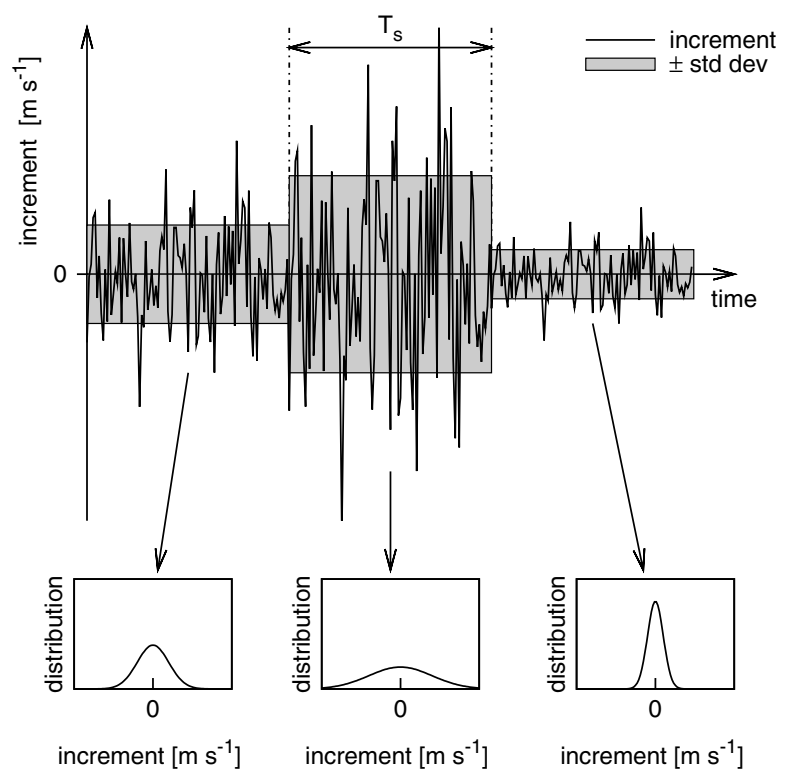

Fig. 2. Schematic sketch of the superstatics approach. The increment series is considered to consist of successive segments of length $T_{s}$ where it can be treated as being normally distributed. Within such a period the mean is considered to be zero and the standard deviation (std dev) is denoted by $1 / \sqrt{\beta}$ so that it gives rise to the $\beta$ series.

in each segment of length $T_{s}$. Figure 2 sketches the parameterisation of the increment series according to the superstatistics approach. For ideal turbulence the $\beta$ series is of a log-normal distribution which means that the logarithm of $\beta$ is normally distributed. The usefulness of the algorithm was demonstrated in Ref. [21], where it was applied to low resolution wind speed recordings at the Florence airport (the velocity was measured every five minutes for one week).

As mentioned above, we apply this algorithm to ABL turbulence with higher resolution $(\nu=8 \mathrm{~Hz})$. It is applied exemplarily to the increment series with $s=8$ of the $24 \mathrm{~h}$ Lammefjord recordings day 191. The temporal scale $T_{s}$ is found to be 116 time steps being equivalent to $14.5 \mathrm{~s}$. Figure 3 shows in the upper left panel the estimated distribution of $\Lambda=\ln \beta$, which is close to a normal distribution. But it has a systematic and statistically significant deviation. In fact, it has a positive skewness. The upper right panel of Fig. 3 depicts the quantile-quantile plot demonstrating that $\Lambda$ is not of a normal distribution. Therefore, we split the $24 \mathrm{~h}$ recording into twelve $2 \mathrm{~h}$ recordings, see the lower panel of Fig. 3. Each sub-series is investigated with respect to superstatistics individually. The $\Lambda$ series of each of the twelve $2 \mathrm{~h}$ recordings were tested for normality using a Kolmogorov-Smirnov test $[22,23]$ whose test variable is plotted in the lowest panel of Fig. 4. Furthermore, this is not only done for $s=8$, but a variety of increment lengths is considered. It is obvious from Fig. 4 that the Castaing hypothesis, i.e., $\Lambda$ is of a Gaussian distribution, can not be rejected with a significance level of $1 \%$. That is, the ABL wind speed fulfils the ideal turbulence hypothesis in each of the twelve $2 \mathrm{~h}$ recordings. The upper and central panel of Fig. 4 show the mean and variance of the $\Lambda$ distribution, respectively, for each of the twelve $2 \mathrm{~h}$ recordings and the variety of increment lengths. They can be interpreted as $\ln \beta_{s}$ and $\lambda_{s}^{2}$, respectively, of the corresponding increment distribution (8). The plot in the central panel demonstrates that the shape parameter $\lambda_{s}^{2}$ approaches zero as the increment length $s$ increases. This is evidence that each of the twelve $2 \mathrm{~h}$ ABL wind speed recordings reflect ideal turbulence whereas the $24 \mathrm{~h}$ recording does not, see Fig. 3.

\section{Stochastic process simulating wind speed}

Motivated by the results of the above mentioned statistical analysis of ABL wind speed, we investigate the geometric $\mathrm{AR}(1)$ process as a suitable model to simulate $\mathrm{ABL}$ wind speed during 
day $191\left(\mathrm{~s}=8, \mathrm{~T}_{\mathrm{s}}=116\right)$

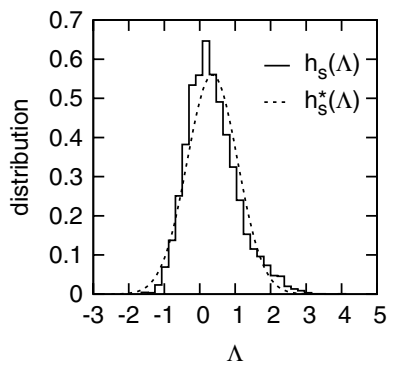

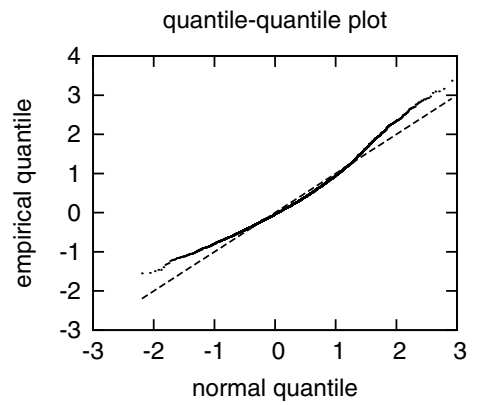

normal quantile

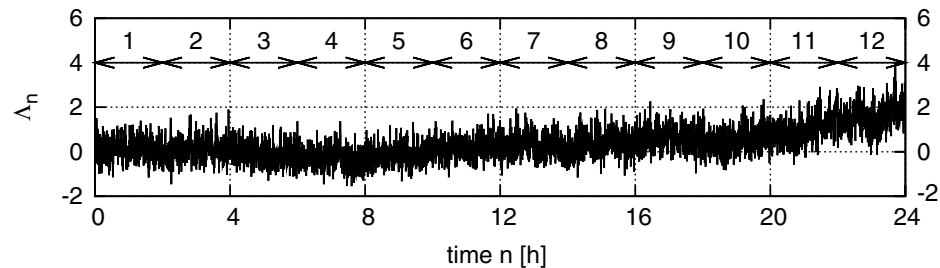

Fig. 3. The top left panel displays the estimated $24 \mathrm{~h} \Lambda$-distribution $h_{s}(\Lambda)$ for $s=8$ of the [5] measurement day 191. $h_{s}^{*}(\Lambda)$ denotes a Gaussian distribution with mean $\langle\Lambda\rangle$ and variance $\left\langle(\Lambda-\langle\Lambda\rangle)^{2}\right\rangle$. The quantile-quantile plot is shown in the upper right panel. The bottom panel displays the $\Lambda$-series for that day. The numbers 1 to 12 illustrate the twelve $2 \mathrm{~h}$ sub-samples which are individually investigated with respect to superstatistics.

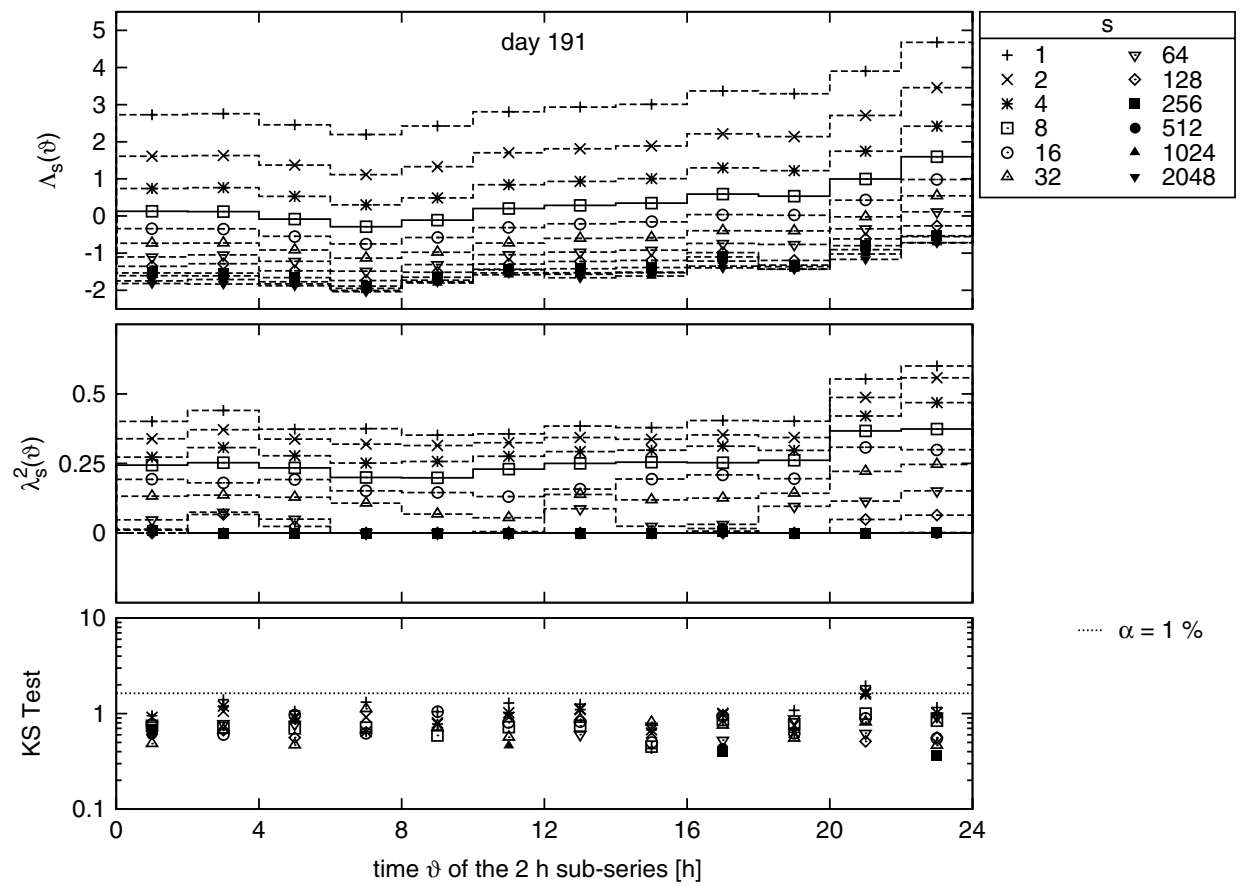

Fig. 4. The top (central) panel shows the mean (variance) of $\Lambda$ for each sub-sample of the data acquired at day 191 at the [5] site and for several $s$. The mean and variance can be identified with the position and shape parameter, respectively. The solid line corresponds to $s=8$ and is therefore related to the graphs in figure 3. The test value of the Kolmogorov-Smirnov test is plotted in the lowest panel. The dashed line corresponds to the critical value for the significance level $\alpha=1 \%$.

an "ideal turbulence phase". It is defined by the recursion formula

$$
\frac{\hat{u}_{n+1}}{\hat{U}}=\left(\frac{\hat{u}_{n}}{\hat{U}}\right)^{a} e^{b \xi_{n}}
$$


for $n=1,2,3, \ldots$ and with $\xi_{n}$ denoting a realisation of white noise. It is characterised by three parameters:

$-\hat{U}$, which has the dimension of velocity and specifies the scale,

$-a$, which determines the degree of correlation between $u_{n+1}$ and $u_{n}$, and

$-b$, which denotes the noise strength.

The process is named geometric $\operatorname{AR}(1)$ process because the logarithm of (9) corresponds to an $\operatorname{AR}(1)$ process. For $|a|<1$ the process $\hat{u}_{n}$ is stationary and of a log-normal distribution with density

$$
\hat{p}(\hat{u})=\frac{1}{\hat{u} \sqrt{2 \pi \lambda^{2}}} \exp \left[\frac{1}{2 \lambda^{2}} \ln ^{2}\left(\frac{\hat{u}}{\hat{U}}\right)\right],
$$

where

$$
\lambda^{2}=\frac{b^{2}}{1-a^{2}}
$$

denotes the variance of $\ln \left(\hat{u}_{n} / \hat{U}\right)$. The auto correlation function [24] reads

$$
\gamma_{\hat{u}}(\tau)=\frac{e^{\lambda^{2} a^{|\tau|}}-1}{e^{\lambda^{2}}-1}
$$

which for small lag decays faster than exponentially and for large lag has an exponential decay.

We show that the process (9) has same statistical properties as ABL wind speed data if

$$
a=1-\varepsilon \quad \text { with } \quad \varepsilon \ll 1 \text { and } b \ll 1 \text {. }
$$

Hence, we can neglect the influence of $a$ in Eq. (9) on the neighbouring time step values $\hat{u}_{n \pm s}$ around $n$ for $0<s \ll 1 / \varepsilon$ and expand any occurring exponential terms in $b$ with respect to $b$.

The fluctuation series (2) of the process $(9)$, denoted by $\hat{f}_{n}^{(m)}$, for a given $m=2 \tilde{m}+1$ which is small enough for the moving average $\overline{\hat{u}}_{n}^{(m)}$ to fluctuate around the expectation value $\hat{U} e^{\lambda^{2} / 2}$ reads

$$
\hat{f}_{n}^{(m)} \sim \overline{\hat{u}}_{n}^{(m)} \times \frac{b}{m} \sum_{l=0}^{\tilde{m}-1}(\tilde{m}-l)\left(\xi_{n-l-1}-\xi_{n+l}\right)
$$

by inserting Eq. (9) and iterations thereof into (2). A full derivation is given in a separate paper [4]. Consequently, the distribution of the fluctuation conditioned on the value $\hat{V}$ of the moving average is given by a symmetric normal distribution with standard deviation $\hat{\alpha}(m) \hat{V}$ where

$$
\hat{\alpha}(m) \sim \frac{b}{2} \sqrt{\frac{m^{2}-1}{3 m}} .
$$

This is in fact one of the desired property a stochastic process must have to simulate wind speed data.

We check that the approximations leading to this result are justified by numerical simulations. We generate $10^{6}$ points using (9) with $a=0.99$ and $b=0.1$. The conditioned histograms of the fluctuation for $m=11$ are shown in Fig. 5. The good agreement with Gaussian shaped curves is evident.

Note that if $m$ is chosen to be large such that $\overline{\hat{u}}^{(m)}$ does not much fluctuate around the expectation value of $u_{n}$, i.e., $\hat{U} e^{\lambda^{2} / 2}$, we have that the fluctuation series corresponds to $\hat{u}_{n}$ shifted by $\hat{U} e^{\lambda^{2} / 2}$. Therefore, the fluctuation distribution is a shifted log-normal. That is, considering the fluctuation over a large period moving average, the process (9) does not reproduce the properties of $\mathrm{ABL}$ turbulence data, whose large period moving average also changes.

Additionally, we need to check whether the increments

$$
\hat{u}_{n ; s}=\hat{u}_{n+s}-\hat{u}_{n}
$$




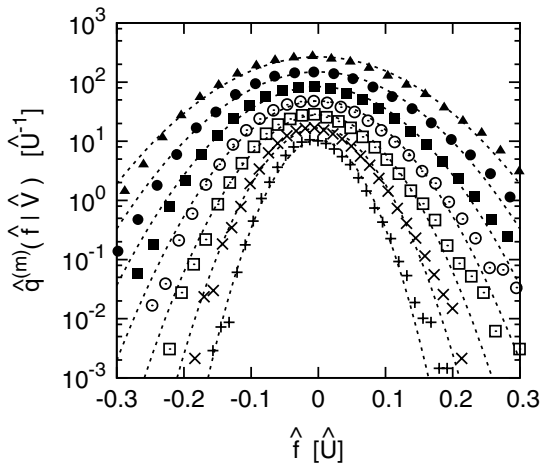

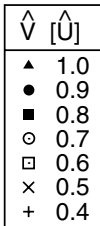

Fig. 5. Fluctuation distributions conditioned on the $m=11$ window mean $\hat{V}$ for a geometric $\operatorname{AR}(1)$ process with $a=0.99$ and $b=0.1$. The dashed lines correspond to symmetric normal distributions. Note that the distributions are shifted against one another in vertical direction for clarity improvement.

of the geometric $\mathrm{AR}(1)$ process as given in (9) and (13) have the same intermittent distribution as ABL wind speed increments. As $s$ can be arbitrarily large, we can not neglect the influence of $a<1$ on $\hat{u}_{n+s}$. In fact, there is no need to do so because the distribution of the random variable (16) can be calculated analytically for arbitrary $a$ with $|a|<1$ and $b$ by the integral

$$
\hat{p}_{s}\left(\hat{u}_{s ; n}\right)=\int \mathrm{d} \hat{u}_{n} \mathrm{~d} \hat{u}_{n+s} \hat{\rho}_{s}\left(\hat{u}_{n}, \hat{u}_{n+s}\right) \delta\left(\hat{u}_{n+s}-\hat{u}_{n}-\hat{u}_{s ; n}\right) .
$$

The joint distribution of $\hat{u}_{n}$ and $\hat{u}_{n+s}$, which is denoted by $\hat{\rho}_{s}\left(\hat{u}_{n}, \hat{u}_{n+s}\right)$, can be determined by iterating (9) $s$ times so that that the increment distribution (17) is given by

$$
\hat{p}_{s}\left(\hat{u}_{n ; s}\right)=\frac{1}{\hat{U} N(s)} \int_{\left|\hat{u}_{n ; s} / \hat{U}\right|}^{\infty} \frac{\mathrm{d} y}{y_{+} y_{-}} \exp \left\{-\frac{\ln ^{2} y_{+}+\ln ^{2} y_{-}-2 a^{s} \ln y_{+} \ln y_{-}}{2 \lambda^{2}\left(1-a^{2 s}\right)}\right\},
$$

where $y_{ \pm}$is abbreviation for $\left(y \pm \hat{u}_{n ; s} / \hat{U}\right) / 2$ and $N(s)=4 \pi \lambda^{2} \sqrt{1-a^{2 s}}$.

We compare the shape of this distribution with the shape of the Castaing distribution (8) with same variance and kurtosis. That is, $\operatorname{Ca}_{s}\left(\hat{\mathrm{u}}_{n ; s}\right)$ with position parameter

$$
\beta_{s}=\frac{1}{2 \hat{U}^{2}\left(1-e^{-\lambda^{2} z(s)}\right)} \sqrt{\frac{1+2 e^{-\lambda^{2} z(s)}+3 e^{-2 \lambda^{2} z(s)}}{6}}
$$

and shape parameter

$$
\Lambda_{s}^{2}=4 \lambda^{2}+\ln \frac{1+2 e^{-\lambda^{2} z(s)}+3 e^{-2 \lambda^{2} z(s)}}{6},
$$

using $z(s)=1-a^{s}$, see [4]. Note that both densities are symmetric and have therefore vanishing odd moments. The integrals (8) and (18) can be computed numerically. Additionally, we estimate the increment distribution from a generated data set $\left(10^{6}\right.$ points $)$ and compare its shape with (18). Figure 6 depicts the increment distribution $\hat{p}_{s}\left(\hat{u}_{n ; s}\right)$ (estimation from data series and analytical solution) and the Castaing distribution $\mathrm{Ca}_{s}\left(\hat{\mathrm{u}}_{n ; s}\right)$ with same variance and kurtosis for a geometric $\operatorname{AR}(1)$ process with $a=0.99$ and $b=0.1$ for two increment lengths: $s=1$ (a) and $s=512(\mathrm{~b})$. The range of the x-axis is approximately \pm 5 times the standard deviation of $\hat{u}_{n ; s}$. It can be inferred from the plots that in good approximation $\hat{p}_{s}\left(\hat{u}_{n ; s}\right) \approx \mathrm{Ca}_{s}\left(\hat{\mathrm{u}}_{n ; s}\right)$.

For ideal turbulence data [11] the shape parameter approaches zero for sufficiently large $s$. However, Eq. (20) states that the shape parameter in the limit of large increment length $s$ of a geometric $\mathrm{AR}(1)$ process takes the value of

$$
\lim _{s \rightarrow \infty} \lambda_{s}^{2}=4 \lambda^{2}+\ln \frac{1+2 e^{-\lambda^{2}}+3 e^{-2 \lambda^{2}}}{6},
$$

which unfortunately is larger than zero. That is, the increment distribution of ideal turbulence data for very large increment length $s$ approaches a symmetric normal distribution whereas the 

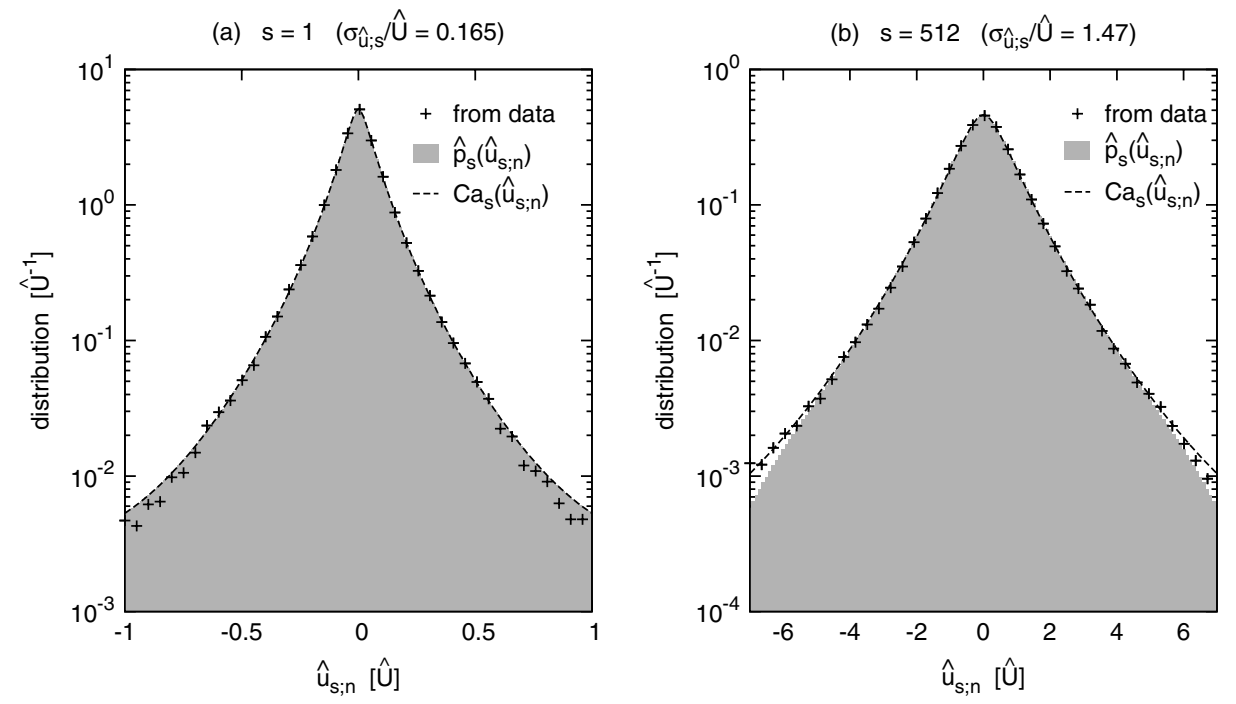

Fig. 6. Increment distributions of a geometric $\operatorname{AR}(1)$ process with $a=0.99$ and $b=0.1$ for $s=1$ and $s=512 . \sigma_{\hat{u} ; s}$ denotes the standard deviation of $\hat{u}_{n ; s}$. The velocity is given in units of $\hat{U}$. The position and shape parameter of the corresponding increment distribution as a function of $s$.

increment distribution of the process (9) is not a symmetric normal distribution in the limit $s \rightarrow \infty$. This is of no surprise because $\hat{u}_{s ; n}$ corresponds for very large $s$ to the difference between two (nearly) independent log-normally distributed random variables which is not a Gaussian distributed random variable.

\section{Conclusion}

It is evident that the mean and the standard deviation of the ABL wind speed data computed on time intervals of hours changes with time. But when considering the fluctuations around a moving window mean, there is a linear relationship between these two quantities. Treating the proportionality factor, the TI, as being constant over $24 \mathrm{~h}$ yields reasonable statistics for the wind speed fluctuation, although one could even reach for a finer time resolution. The wind velocity can be treated as a superposition of a random variable reflecting the current mean wind speed with a normally distributed random variable with vanishing mean and a standard deviation being proportional to the current mean wind speed. As a cross-check, if the mean speed does not change, ABL wind speed has the same statistics as air speed measured in stationary laboratory turbulence: a nearly Gaussian distribution.

Moreover, a time series reflecting ABL wind speed turbulence can be treated as consisting of subsequent sub-series reflecting ideal turbulence. That is, the parameters of the increment distribution change with time whereas within such a phase they can be treated as being constant. The superstatics approach is capable of resolving their time dependence.

The geometric $\mathrm{AR}(1)$ process (9) with exponent $a \lesssim 1$ and the noise strength $b \ll 1$ is a reasonable and very simple stochastic process for simulating ABL wind speed during an "ideal turbulence phase". It has the same fluctuation distribution conditioned on the value $V$ of the moving average as observed in ABL wind speed data: a symmetric Gaussian with a standard deviation being proportional to $V$. Additionally, it has nearly the same increment statistics as observed in ABL wind speed data: a Castaing distribution. Even the shape parameter decreases as the increment length $s$ becomes larger. However, this model does not provide a clear crossover between a fat-tailed distribution for small $s$ and a Gaussian distribution for large $s$.

The authors would like to thank the Germany's Federal Ministry for Education and Research (BMBF) for funding this study under grant number 03SF0314. The presented results are part of the project "Statistical analysis and stochastic modelling of turbulent gusts in surface wind". 


\section{References}

1. R. Thuillier, U.O. Lappe, J. Appl. Meteor. 3, 299 (1964)

2. T. Burton, D. Sharpe, N. Jenkins, E. Bossanyi, Wind Energy Handbook (John Wiley, 2004)

3. T. Laubrich, F. Ghasemi, J. Peinke, H. Kantz [arXiv:0811.3337] (2008)

4. T. Laubrich, H. Kantz (2009) (to be published)

5. Lammefjord, Lammefjord data obtained from the Ris $\varnothing$ National Laboratory in Denmark, http://www.risoe.dk/vea, through http://www.winddata.com. (1987)

6. J.M. Wallace, P.V. Hobbs, Atmospheric Science (Academic Press Elsevier, 2006)

7. U. Frisch, Turbulence (Cambridge University Press, 1995)

8. S.B. Pope, Turbulent Flows (Cambridge University Press, 2000)

9. G. Taylor, Proc. R. Soc. London Ser. A 164, 476 (1938)

10. R. Stull, An Introduction to Boundary Layer Meteorology (Springer, 1999)

11. C. Renner, J. Peinke, R. Friedrich, J. Fluid Mech. 433, 383 (2001)

12. A.N. Kolmogorov, Dokl. Akad. Nauk SSSR 30, 299 (1941)

13. A.N. Kolmogorov, Dokl. Akad. Nauk SSSR 32, 16 (1941)

14. A.N. Kolmogorov, J. Fluid Mech. 13, 82 (1962)

15. B. Castaing, Y. Gagne, E.J. Hopfinger, Physica D 46, 177 (1990)

16. F. Boettcher, C. Renner, H.P. Waldl, J. Peinke, Bound.-Layer Meteor. 108, 163 (2003)

17. F. Boettcher, S. Barth, J. Peinke, Stoch. Environ. Res. Risk Assess. 21, 299 (2007)

18. C. Beck, E.G.D. Cohen, Physica A 322, 267 (2003)

19. C. Beck, E.G.D. Cohen, H. Swinney, Phys. Rev. E 72, 056133 (2005)

20. S.M.D. Queiros, Physica A 385, 191 (2007)

21. C. Beck, E.G.D. Cohen, S. Rizzo, Europhysics News 36, 189 (2005)

22. W. Daniel, Applied Nonparametric Statistics (PWS-Kent, 1990)

23. L. Miller, J. Amer. Statist. Assoc. 51, 111 (1956)

24. C.W.J. Granger, P. Newbold, J. R. Statist. Soc. Ser. B 38, 189 (1976) 